\title{
Annealing-induced changes of the magnetic anisotropy of (Ga,Mn)As epilayers
}

\author{
V. Stanciu ${ }^{1, *}$ and P. Svedlindh ${ }^{1}$ \\ ${ }^{1}$ Department of Engineering Sciences, Uppsala University, Box 534, SE-751 21 Uppsala, Sweden
}

(Dated: June 24, 2018)

\begin{abstract}
The dependence of the magnetic anisotropy of As-capped (Ga,Mn)As epilayers on the annealing parameters - temperature and time - has been investigated. A uniaxial magnetic anisotropy is evidenced, whose orientation with respect to the crystallographic axes changes upon annealing from [110] for the as-grown samples to [110] for the annealed samples. Both cubic an uniaxial anisotropies are tightly linked to the concentration of charge carriers, the magnitude of which is controlled by the annealing process.

PACS numbers: 75.50.Pp, 75.30.Gw, 75.70.-i
\end{abstract}

Considerable experimental and theoretical efforts have recently been devoted to the study of III-V diluted magnetic semiconductors (DMS) due to their potential implementation as spintronic devices [1]. The high ferromagnetic transition temperature of (Ga,Mn)As has made this compound one of the most investigated III-V DMS, being regarded as the prototype of this new class of materials 2]. It is well established nowadays that the ferromagnetic interaction between the magnetic ions is mediated by the charge carriers, holes in this case, and that point defects such as As antisites $\left(\mathrm{As}_{G a}\right)$ and $\mathrm{Mn}$ interstitials $\left(\mathrm{Mn}_{I}\right)$ play a crucial role in determining the magnetic properties of $(\mathrm{Ga}, \mathrm{Mn}) \mathrm{As}$ 3, 4, 5]. Optimal annealing experiments have proved that the highly mobile $\mathrm{Mn}_{I}$ defects 3 outdiffuse to and are passivated at the free surface [5, [6] and that in all successful annealings the $\mathrm{Mn}_{I}$ concentration is higher than the concentration of $\mathrm{As}_{G a}$. The removal of $\mathrm{Mn}_{I}$ from the bulk of $(\mathrm{Ga}, \mathrm{Mn})$ As leads to an increase of the ferromagnetic transition temperature $\left(T_{C}\right)$, carrier concentration and average manganese magnetic moment. However, one particular aspect of the magnetic properties is not fully understood and this concerns the magnetic anisotropy. Experimentally it is found that a strong uniaxial (UA) contribution to the overall magnetic anisotropy appears along the [110] direction 7, 8, 9]. Although the $(\mathrm{Ga}, \mathrm{Mn})$ As structure is tetragonally distorted due to its lattice mismatch with the GaAs substrate, structural characterization commonly shows that the epilayers are coherently strained to the substrate 10. and this holds even for very large thicknesses of the order of a few $\mu \mathrm{m}[8]$. Theoretical investigations explain fairly well the influence of the biaxial strain and hole concentration on the magnetic anisotropy 11, 12. However, based on symmetry arguments the tetragonal distortion cannot account for the appearance of the uniaxial anisotropy. Anisotropy measurements on as-grown samples of different thicknesses [8] and on annealed-and-etched samples [9] have shown that the UA contribution does not have a surface or interface origin. Instead, it has been suggested by Sawicki et al. [9] that the UA originates from a small

*Electronic address: victor.stanciu@angstrom.uu.se
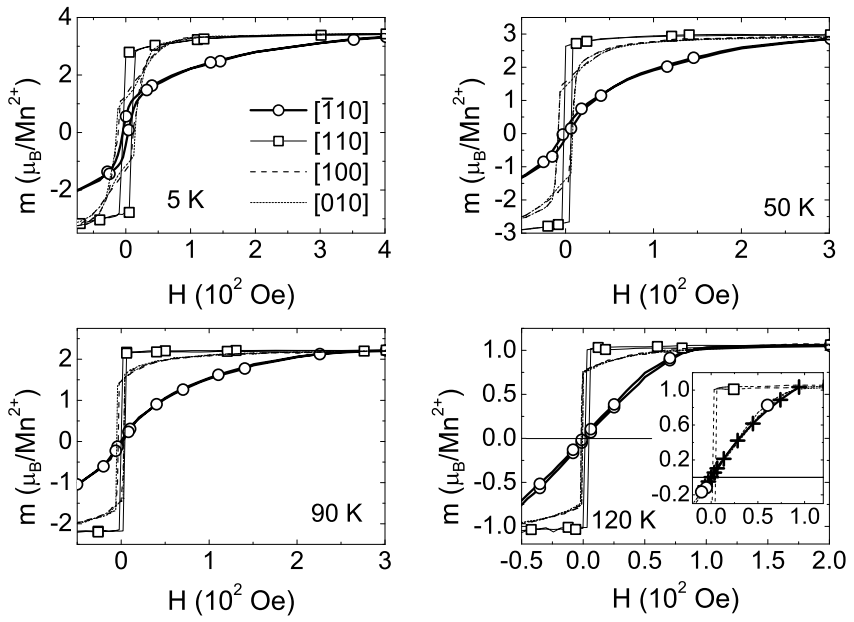

FIG. 1: Field dependence of the magnetization recorded at different temperatures for a $1000 \AA$ thick sample annealed at $240{ }^{\circ} \mathrm{C}$ for $10 \mathrm{~h}$. The crosses in the inset of the ' $120 \mathrm{~K}$ ' pane represent a fit to the hard axis magnetization as discussed in the text.

trigonal distortion $\epsilon_{x y} \neq 0$; using the $p-d$ Zener model of the ferromagnetism they estimate that $\epsilon_{x y}=0.05 \%$ is enough to explain the experimentally observed UA. The microscopic origin of the trigonal distortion could be surface As-dimerization, resulting in surface reconstruction and an inequivalence between the [110] and [110] directions [7].

The $\mathrm{Ga}_{1-x} \mathrm{Mn}_{x}$ As $(x \sim 0.06)$ samples were grown on (001) GaAs substrates by low-temperature molecular beam epitaxy at a growth temperature $\left(T_{g}\right)$ of about $230{ }^{\circ} \mathrm{C}$. Further details on the sample preparation are given elsewhere [13]. For an efficient annealing, a rather thick capping layer of amorphous As was deposited on top of $(\mathrm{Ga}, \mathrm{Mn})$ As. Pieces of the as-grown samples were annealed in air at different annealing temperatures $\left(T_{a}\right)$ and for different annealing times $\left(t_{a}\right)$. We have shown that the dependence of $T_{C}$ on the annealing time has a peculiar variation due to the presence of the amorphous cap [6]. Depending on the 'position' of $T_{a}$ with respect to $T_{g}, T_{C}$-peaks appear at certain values of $t_{a}\left(t_{a}^{\text {peak }}\right)$. In this study we focus only on $T_{a}$ either lower $\left(215{ }^{\circ} \mathrm{C}\right)$ or 
TABLE I: $T_{C}$ values for the $1000 \AA$ and $300 \AA$ thick samples annealed at $215{ }^{\circ} \mathrm{C}$ and $240{ }^{\circ} \mathrm{C}$ for different $t_{a}$. The as-grown samples are denoted by ' $a$ '.

\begin{tabular}{c|c|ccc|ccc|}
\hline \hline & \multicolumn{7}{|c|}{$t=1000 \AA$} \\
\hline$T_{a}$ & $\mathrm{a}$ & \multicolumn{3}{|c|}{$215^{\circ} \mathrm{C}$} & \multicolumn{3}{c|}{$240{ }^{\circ} \mathrm{C}$} \\
\hline$t_{a}$ & & $3 \mathrm{~h}$ & $7 \mathrm{~h}$ & $10 \mathrm{~h}$ & $1 \mathrm{~h}$ & $4 \mathrm{~h}$ & $10 \mathrm{~h}$ \\
\hline$T_{C}(\mathrm{~K})$ & 76 & 127 & 136 & 132 & 127 & 145 & 130 \\
\hline \hline & \multicolumn{7}{|c|}{$t=300 \AA$} \\
\hline$T_{a}$ & $\mathrm{a}$ & \multicolumn{3}{|c|}{$215{ }^{\circ} \mathrm{C}$} & \multicolumn{3}{c|}{$240{ }^{\circ} \mathrm{C}$} \\
\hline$t_{a}$ & & $1 \mathrm{~h}$ & $5 \mathrm{~h}$ & $9 \mathrm{~h}$ & $3 \mathrm{~h}$ & $4 \mathrm{~h}$ & $10 \mathrm{~h}$ \\
\hline$T_{C}(\mathrm{~K})$ & 82 & 127 & 136 & 131 & 134 & 122 & 126 \\
\hline \hline
\end{tabular}

higher $\left(240^{\circ} \mathrm{C}\right)$ than $T_{g}$. The annealing times are chosen relative to the $t_{a}^{\text {peak }}$ position in order to cover $t_{a}$-regions below and above $t_{a}^{\text {peak }}[$ ] .

The magnetic anisotropy of the samples was assessed from field-dependent measurements of the magnetization recorded in a QuantumDesign MPMS-XL SQUID magnetometer. For a detailed investigation of the magnetic anisotropy, hysteresis loops were measured along four different crystallographic directions, namely [100], [110], [010] and [110], and at different temperatures, both for the as-grown and annealed samples. An example of such hysteresis loops is shown in Fig. 11 for a $1000 \AA$ thick sample annealed for $10 \mathrm{~h}$ at $240{ }^{\circ} \mathrm{C}$. The $T_{C}$ was derived from the temperature dependence of the magnetization, $M(T)$, as the onset of the FM order; $T_{C}$ values for the different samples are summarized in Table [1] The free energy density, considering a cubic, an uniaxial and a Zeeman term, can be written as $e=K_{c} \sin ^{2}(\theta) \cos ^{2}(\theta)+$ $K_{u} \sin ^{2}(\theta-\pi / 4)-\mu_{0} M_{s} H \cos \left(\theta-\alpha_{H}\right)$, where $\theta$ and $\alpha_{H}$ give the orientation of the saturation magnetization $\left(M_{s}\right)$ and applied field with respect to the [100] direction, arbitrarily chosen as a reference axis. In our measurements, $\alpha_{H}$ therefore assumes the following values $0, \pi / 4, \pi / 2$ and $3 \pi / 4$. Minimization of the free energy with respect to the angle $\theta$, and for particular values of $\alpha_{H}$, gives relations between the applied field and the magnetization as in Eq. 1] $\left(\alpha_{H}=3 \pi / 4\right)$.

$$
\frac{2 K_{c}}{\mu_{0} M_{s}}\left[2\left(\frac{M}{M_{s}}\right)^{3}-\frac{M}{M_{s}}\right]+\frac{2 K_{u}}{\mu_{0} M_{s}} \frac{M}{M_{s}}=H
$$

The anisotropy constants, $K_{u}$ and $K_{c}$, are found from fits to the measured hard-axis magnetizations, with the hard-axis corresponding to the [110] and [110] directions for the as-grown and annealed samples, respectively. An example of such a fit is shown in the inset of the ' 120 K' pane of Fig. 1 (the fit is marked by crosses). One should stress that errors stemming from determination of the sample volume (thickness and area) directly influence the value of the saturation magnetization and as a result rather large relative errors of $5 \div 10 \%$ are introduced when calculating the anisotropy constants.

Figures 2 and 3 show the temperature dependence of the anisotropy constants for the $1000 \AA$ and $300 \AA$ thick
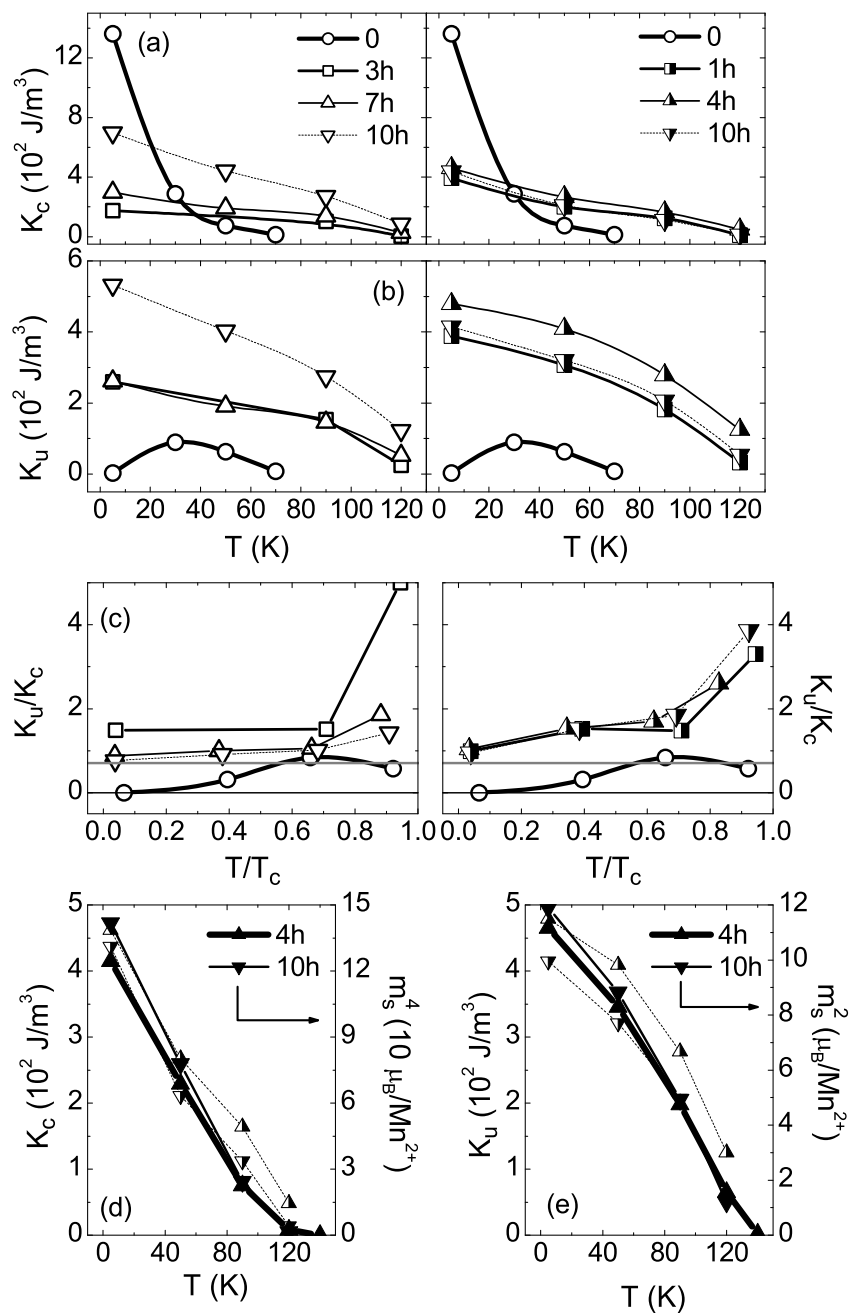

FIG. 2: (a) and (b) $K_{c}$ and $K_{u}$ vs. temperature for a $1000 \AA$ thick sample annealed at $215{ }^{\circ} \mathrm{C}$ (empty symbols, left hand panes) and $240{ }^{\circ} \mathrm{C}$ (half-filled symbols, right hand panes). (c) The ratio $K_{u} / K_{c}$ as a function of the reduce temperature $T / T_{c}$ for $T_{a}=215{ }^{\circ} \mathrm{C}$ (left hand pane) and $T_{a}=240{ }^{\circ} \mathrm{C}$ (right hand pane). (d) $K_{c}$ (dashed lines) and $m_{s}^{4}$ (solid lines) vs. temperature $\left(T_{a}=240{ }^{\circ} \mathrm{C}\right)$. (e) $K_{u}$ (dashed lines) and $m_{s}^{2}$ (solid lines) vs. temperature $\left(T_{a}=240^{\circ} \mathrm{C}\right)$.

films, respectively. The relative contribution of the two anisotropies to the overall anisotropy is given by the ratio $K_{u} / K_{c}$. This ratio is plotted in Figs. 2 (c) and 3 (c) for the two annealing temperatures. If $K_{u} / K_{c} \geqslant 1$, the EA is pinned along [110]; if $0 \leqslant K_{u} / K_{c}<1$, the EA assumes intermediate directions between [110] and [100]; if $1 / \sqrt{2} \leqslant K_{u} / K_{c}<1$, the EA is closer to [110] and vice versa (the grey line in the figures corresponds to $\left.K_{u} / K_{c}=1 / \sqrt{2}\right)$. In the limit case of a cubic anisotropy, i.e. EA along [100], $K_{u}$ tends to zero. All these situations can be easily seen in the hysteresis loops. For instance, in Fig. 11 at $5 \mathrm{~K}, K_{u} / K_{c}=0.9$, thus the EA is very close to [110], while at elevated temperatures, as $K_{u} / K_{c}$ exceeds 1 , square hysteresis loops are obtained with $M_{r}^{[110]} \simeq M_{s}$, 

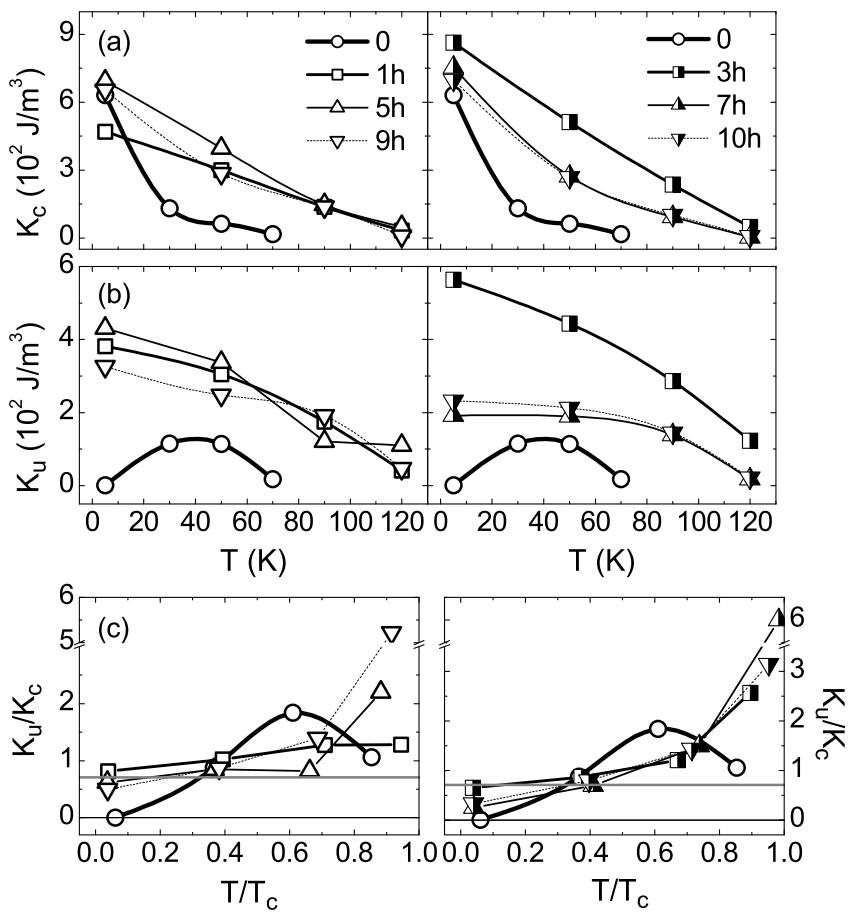

FIG. 3: (a) and (b) $K_{c}$ and $K_{u}$ vs. temperature for a $300-\AA$ thick sample annealed at $215{ }^{\circ} \mathrm{C}$ (empty symbols, left hand panes) and $240{ }^{\circ} \mathrm{C}$ (half-filled symbols, right hand panes). (c) The ratio $K_{u} / K_{c}$ as a function of the reduced temperature $T / T_{c}$ for $T_{a}=215{ }^{\circ} \mathrm{C}$ (left hand pane) and $T_{a}=240{ }^{\circ} \mathrm{C}$ (right hand pane).

where $M_{r}$ is the remanence magnetization. In Fig. 2 (d) and (e), one may notice that $K_{c}$ and $K_{u}$ exhibit a temperature dependence in agreement with that of $m_{s}^{4}$ and $m_{s}^{2}$, respectively, as expected for the cubic and uniaxial anisotropy terms.
For the as-grown samples we find that at low temperatures the uniaxial contribution is negligible as compared to cubic one; the ratio $K_{u} / K_{c}$ approaches zero in this case, and thus an equivalence between the $\langle 100\rangle$ and $\langle 110\rangle$ axes is found in the hysteresis loops. As the temperature increases, $K_{c}$ has a rapid fall-off and an uniaxial contribution appears with the EA oriented (roughly) along [110]. It should be noted, using the free energy expression for the magnetization given above, that the extracted value of $K_{u}$ is negative for the as-grown samples. However, in Figs. 2 (b) and 3] (b) the magnitude of $K_{u}$ is plotted instead. Furthermore, the temperature dependence of $K_{u}$ is similar for both the $1000 \AA$ and $300 \AA$ thick samples, whereas $K_{c}$ is larger for the 1000 $\AA$ sample than for the $300 \AA$ sample.

A 90-degree rotation of the UA takes place upon annealing. This rotation occurs even for very short $t_{a}$ signaling a strong correlation between the UA direction and the hole concentration as pointed out recently by Sawicki et al. [9]. For $T_{a}$ close to $T_{g}$ short annealing times are needed to readily deplete the bulk of $(\mathrm{Ga}, \mathrm{Mn}) \mathrm{As}$ from $\mathrm{Mn}_{I}$, and thus a large increase of the hole concentration is expected [6]. Another manifestation of the close connection between magnetic anisotropy and carrier concentration is the variation of the anisotropy constants with $t_{a}$ since both $K_{c}$ and $K_{u}$ exhibit maxima at $t_{a}=t_{a}^{\text {peak }}$; a maximum in hole concentration at $t_{a}=t_{a}^{\text {peak }}$ was inferred from the annealing experiments reported in Ref. [6].

In summary, we have shown that both cubic and uniaxial anisotropies appearing in (Ga,Mn)As are influenced by changes in the hole concentration, which in turn is controlled by the different annealing parameters.

The Swedish Foundation for Strategic Research (SSF) and the Swedish Research Council (VR) are acknowledged for financial support.
[1] S. A. Wolf, D. D. Awschalom, R. A. Buhrman, J. M. Daughton, S. von Molnar, M. L. Roukes, A. Y. Chtchelkanova, and D. M. Treger, Science 294, 1488 (2001).

[2] A. H. MacDonald, P. Schiffer, and N. Samarth, Nature Mater. 4, 195 (2005).

[3] K. M. Yu, W. Walukiewicz, T. Wojtowicz, I. Kuryliszyn, X. Liu, Y. Sasaki, and J. K. Furdyna, Phys. Rev. B 65, 201303(R) (2002).

[4] K. C. Ku, S. J. Potashnik, R. F. Wang, S. H. Chun, P. Schiffer, N. Samarth, M. J. Seong, A. Mascarenhas, E. Johnston-Halperin, R. C. Myers, et al., Appl. Phys. Lett. 82, 2302 (2003).

[5] K. W. Edmonds, P. Boguslawski, K. Y. Wang, R. P. Campion, S. N. Novikov, N. R. S. Farley, B. L. Gallagher, C. T. Foxon, M. Sawicki, T. Dietl, et al., Phys. Rev. Lett. 92, 037201 (2004).

[6] V. Stanciu, O. Wilhelmsson, U. Bexell, M. Adell, J. Sadowski, J. Kanski, P. Warnicke, and P. Svedlindh, condmat/0505040.
[7] U. Welp, V. K. Vlasko-Vlasov, X. Liu, J. K. Furdyna, and T. Wojtowicz, Phys. Rev. Lett. 90, 167206 (2003).

[8] U. Welp, V. Vlasko-Vlasov, A. Menzel, H. You, X. Liu, J. Furdyna, and T. Wojtowicz, Appl. Phys. Lett. 85, 260 (2004).

[9] M. Sawicki, K.-Y. Wang, K. W. Edmonds, R. P. Campion, C. R. Staddon, N. R. S. Farley, C. T. Foxon, E. Papis, E. Kaminska, A. Piotrowska, et al., Phys. Rev. B 71, 121302 (2005).

[10] G. M. Schott, W. Faschinger, and L. W. Molenkamp, Appl. Phys. Lett. 79, 1807 (2001).

[11] T. Dietl, H. Ohno, and F. Matsukura, Phys. Rev. B 63, 195205 (2001).

[12] M. Abolfath, T. Jungwirth, J. Brum, and A. H. MacDonald, Phys. Rev. B 63, 054418 (2001).

[13] M. Adell, L. Ilver, J. Kanski, V. Stanciu, P. Svedlindh, J. Sadowski, J. Z. Domagala, F. Terki, C. Hernandez, and S. Charar, Appl. Phys. Lett. 86, 112501 (2005). 